Published in final edited form as:

Lung Cancer. 2006 October ; 54(Suppl 1): S1-60.

\title{
Introduction
}

\author{
Gerold Bepler $^{1}$ and Nick Thatcher ${ }^{2}$ \\ 1 Gerold Bepler, MD, PhD, H. Lee Moffitt Cancer Center and Research Institute, Tampa, FL, USA \\ 2 Nick Thatcher, Professor of Oncology, Department of Medical Oncology, Christie Hospital NHS Trust, \\ Manchester, UK
}

Lung cancer is widely recognised as the leading cause of cancer-related mortality in the Western world. Malignant pleural mesothelioma (MPM) is a particularly aggressive form of asbestos-related cancer in which early attempts at treatment have yielded few results. However, with the introduction of new, more effective treatment modalities, the scepticism that previously surrounded this devastating neoplasm is gradually receding. The combination of pemetrexed plus cisplatin in first-line therapy has had a significant impact, leading to improved survival and offering patients with MPM the potential for an improved quality of life. Research focus is now turning to neoadjuvant, second-line and maintenance chemotherapy for MPM.

Non-small cell lung cancer (NSCLC) accounts for approximately $80 \%$ of thoracic malignancies, and the large majority of patients present with disease that is beyond the scope of surgical cure. These patients are, therefore, candidates for palliative chemotherapy, which has been the subject of intensive investigation in recent years. Platinum-based combination chemotherapy has been identified as the standard of care for the first-line treatment of NSCLC, leading to an increase in survival, improved quality of life and a need for second- and thirdline treatment, which currently represents a particularly active area of research. As a result, the available number of therapeutic options for previously treated NSCLC patients is growing, and individual patient characteristics and side-effect profiles are increasingly influencing the therapeutic choices made by physicians.

A number of new targeted therapies are currently being investigated, and continued research is needed to determine the role of these agents in the treatment of thoracic malignancies. One particularly promising area of interest is the use of molecular markers to identify subgroups of patients who have an increased likelihood of a treatment response based on their genetic profile. Since tailored treatment has the potential to improve patient outcome dramatically, pharmacogenomics will no doubt play an important role in the future of lung cancer therapy.

\section{Scope of the supplement}

This supplement to Lung Cancer describes the current standard of care in NSCLC and mesothelioma, and discusses the application and integration of new agents, novel therapeutic approaches and pharmacogenomics in the treatment of thoracic cancers.

In the first article on the pharmacogenomics of NSCLC, Dr Bepler outlines the available evidence on the use of genetic markers to predict patient response to cytotoxic drugs. Steps leading to the realisation that $R R M 1$ gene expression can influence patient outcome and response to chemotherapy are outlined, and the potential implications of this for treating

Publisher's Disclaimer: This is a PDF file of an unedited manuscript that has been accepted for publication. As a service to our customers we are providing this early version of the manuscript. The manuscript will undergo copyediting, typesetting, and review of the resulting proof before it is published in its final citable form. Please note that during the production process errors may be discovered which could affect the content, and all legal disclaimers that apply to the journal pertain. 
NSCLC are discussed. Unpublished results from a groundbreaking study suggesting that genetic pre-selection of patients can greatly improve survival in NSCLC are described, as is the potential use of immunohistochemistry in the identification of genetic markers.

In the second article, Dr Stahel focuses on MPM, which generally has a poor prognosis and is refractory to conventional therapy. A rise in the incidence of this important disease is predicted over the next decade. However, the realisation that patients can benefit from systemic chemotherapy and may respond to multimodality approaches provides hope for the management of this epidemic. The antifolate pemetrexed has shown particular promise in the treatment of MPM, and the current applications and ongoing investigations of this agent in various settings are reviewed.

Returning to NSCLC, Dr Vansteenkiste explains that several therapeutic options are now available for the second-line treatment of this malignancy. The 21 st century has witnessed the achievement of improved response rates and median and 1-year survival times following second-line treatment of NCSLC. In light of the increasing number of available active agents, a rational, evidence-based approach to first-, second- and third-line therapy is proposed in this article. In addition, observations of the specific activity of targeted agents in certain patient subgroups are outlined.

The next article by Dr Crinò observes that an efficacy plateau appears to have been reached in first-line chemotherapy for advanced or metastatic NSCLC and that third-generation platinumbased combinations now represent the standard of care. Research efforts have, therefore, been directed towards the identification of new first-line regimens that maintain the same level of efficacy as standard platinum-based combinations but offer a better toxicity profile. Evidence indicates that pemetrexed has the potential to play a key role in this regard, and results are presented demonstrating the safety and efficacy of this agent, combined with oxaliplatin or carboplatin, in the first-line treatment of locally advanced or metastatic NSCLC. In addition, bevacizumab is highlighted as the first molecular targeted agent to have demonstrated a survival advantage in combination with chemotherapy for the first-line treatment of advanced non-squamous NSCLC, and ZD6474 is identified as another promising new targeted agent in this setting.

In the concluding article, Dr Thatcher continues this theme by describing the potential applications of novel targeted agents in NSCLC. Such agents appear to fit into current therapy for NCSLC at various stages, either as monotherapy or in combination with other agents depending on the setting, and may be of particular benefit in certain patient subgroups. Current challenges related to the integration of these agents into existing strategies are discussed, including how to sequence treatments in order to maximise the therapeutic benefit achieved with subsequent treatment approaches.

This supplement aims to provide the reader with a comprehensive overview of both currently available and investigational treatment regimens, and highlights issues associated with the introduction of novel agents for treating lung cancer. Promising observations related to this challenging malignancy are increasingly being reported in the literature, and the available evidence indicates that gradual but definite progress is being made to improve the care of patients with thoracic tumours.

\section{Acknowledgements}

Eli Lilly is gratefully acknowledged for providing an educational grant to support this supplement and its publication, and all participants are thanked for their valuable contributions. 